\section{Localisation of lysozyme mRNA in rheumatoid synovial membrane by in situ hybridisation}

SIR, We were interested to read the article by Konttinen et al, ${ }^{1}$ in which our previous demonstration of the presence of lysozyme in lysosomal bodies of type A cells was confirmed. We remain puzzled by the suggestion that in situ hybridisation was necessary to show that the lysozyme did not represent material phagocytosed by these cells and that it could have originated from mononuclear phagocytes in the synovial fluid or cartilage. If such phagocytosed lysozyme were present in lysosomes it would presumably be indistinguishable from the lysozyme of these bodies themselves. Dr Konttinen has shown the presence of mRNA for lysozyme in cells lining the synovial surface but has no double labelling or parallel ultrastructural studies to confirm his suggestion that these are type $\mathbf{A}$ synoviocytes.

Our immunogold ultrastructural study clearly shows the localisation of lysozyme to primary lysosomes. ${ }^{2}$ Lysozyme was often localised to phagocytic vacuoles containing iron, the presence of which was confirmed by $x$ ray microprobe analysis. The possibility of secondary uptake of lysozyme is unlikely as it was localised to primary rather than secondary lysosomes.

The London Hospital Medical College, Arthritis and Rheumatism Council Building, 25-29 Ashfield Street, London E1 1AD

\section{References}

1 Konttinen Y T, Bergroth V, Kulomaa M, et al. Localisation of lysozyme mRNA in rheumatoid synovial membrane by in situ hybridisation. Ann Rheum Dis 1989; 48: 912-7.

2 Mapp P I, Revell P A. Ultrastructural localisation of muramidase in the human synovial membrane. Ann Rheum Dis 1987; 46: 30-7.

\section{Reflex sympathetic dystrophy associated with large cell lung carcinoma}

SIR, We noted the report of Prowse et al in the Annals ${ }^{1}$ and wish to report a further case of reflex sympathetic dystrophy associated with a large cell lung carcinoma. In our case there were fewer early vasomotor features. The underlying neoplasm was not evident for nearly three years after the initial presentation.

A 69 year old retired fisherman noted right shoulder and wrist pain after a minor fall on his outstretched right arm. His pain gradually increased in intensity over the next six months, becoming worst over his right shoulder. Pain was less while resting and made worse by using his arm. Because $x$ rays showed a moderate degree of cervical spondylosis he was considered to have symptomatic cervical spondylosis.

His pain became more severe a year after the onset of his symptoms. At 16 months he had a normal bone scan and cervical myelogram performed. As his right shoulder movements were markedly restricted he had intra-articular steroid injections followed by manipulation under anaesthesia, which did not help. He continued to complain of severe pain over the next year, which was unrelieved by non-steroidal anti-inflammatory drugs.

Two and a half years after the onset of his symptoms he had a myocardial infarction. He had been a 30 cigarette a day smoker until eight years before this. Chest $x$ rays at this time showed no evidence of an apical mass or bony destruction. Over the next three months the right shoulder and arm pain became more severe, causing sleep disturbance. He noted also paraesthesiae in his fingers, a reduced grip power, and that, on occasion, his right hand was colder than the left. He had lost $6 \mathrm{~kg}$ over the two years before myocardial infarction and $13 \mathrm{~kg}$ in the three months before he was referred to the rheumatology department for assessment.

Examination showed a cachetic man with a cool, mottled, right arm, marked intrinsic hand muscle wasting, and severe right shoulder movement restriction. There was increased local heat over the right hemithorax compared with the left side. Chest $x$ ray showed some opacity in the right apical region not previously visible (even on subsequent review) on his $x$ ray three months earlier. In addition, there were gross destructive changes visible in the second right rib. Computed tomography confirmed the mass and rib destruction, while bone scan was again normal. Cytology showed a probable large cell undifferentiated carcinoma. Palliative radiotherapy improved his symptoms, but he died two and a half months later.

Doury has made the point that, though common, reflex sympathetic dystrophy is often unrecognised. ${ }^{2}$ The long duration of symptoms in our case and the unusual association highlight the need to continue to look for an underlying lesion in patients with reflex sympathetic dystrophy that does not respond to treatment.

Mater Misericordiae Hospital, Dublin 7

MARION REGAN

DARRAGH FOLEY-NOLAN GERALDINE MCCARTHY ROBERT J COUGHLAN

\section{References}

1 Prowse M, Higgs C M B, Forrester-Wood C. McHugh N. Ann Rheum Dis 1989; 48: 339-41.

2 Doury $P$. Review of algodystrophy. Reflex sympathetic dystrophy. Clin Rheumatol 1988; 7: 173-80. 